\title{
Anatomical Planes in Rectal Cancer Surgery
}

\section{Rektum Kanser Cerrahisinde Anatomik Planlar}

\author{
(1) Halil İbrahim Açar, (1) Mehmet Ayhan Kuzu \\ Ankara University Faculty of Medicine, Department of General Surgery, Ankara, Turkey
}

\section{HIIIIII| ABSTRACT}

This review outlines important anatomical landmarks not only for rectal cancer surgery but also for pelvic exentration.

Keywords: Anorectal anatomy, pelvic anatomy, surgical anatomy of rectum

\section{HIIIIIII ÖZ}

Pelvis anatomisini derleme halinde özetleyen bu makale rektum kanser cerrahisi ve pelvik ezantrasyon için önemli topografik noktaları gözden geçirmektedir.

Anahtar Kelimeler: Anorektal anatomi, pelvik anatomi, rektumun cerrahi anatomisi

\section{Introduction}

\section{Pelvic Anatomy}

It is essential to know the pelvic anatomy because of the intestinal and urogenital complications that may develop after the surgical procedures applied to the pelvic region. The pelvis, encircled by bone tissue, is surrounded by the main vessels, ureters, and autonomic nerves. Success in the surgical treatment of pelvic organs is only possible with a good knowledge of the embryological development of the pelvic structures. Rectal cancer is a common disease both in our country and in the world. However, technical problems in surgical treatment are important because of postoperative complications and recurrence problems. Modern imaging studies have shown that the spread of the disease is related to the embryological development of the rectum. For this reason, dissection for curative surgery in rectal cancer can only be achieved with a good knowledge of the embryological and anatomical planes. ${ }^{1}$

\section{Surgical Anatomy of the Rectum}

The rectum extends from the promontory to the anal canal and is approximately $12-15 \mathrm{~cm}$ long. It fills the sacral concavity and ends with an anal canal $2-3 \mathrm{~cm}$ anteroinferior to the tip of the coccyx. The rectum contains three folds in the coronal plane laterally. The upper and lower are convex to the right, and the middle is convex to the left. The middle fold is aligned with the peritoneal reflection. Intraluminal projections of the lower boundaries of these folds are known as Houston's valves. Unlike the sigmoid colon, taenia, epiploic appendices, and haustra are absent in the rectum. The upper $1 / 3$ part is covered with peritoneum anteriorly and laterally, and the middle $1 / 3$ part is covered anteriorly. The lower $1 / 3$ part is entirely extraperitoneal. The rectum is considered to be roughly divided into three sections as upper, middle, and lower rectum. Although these are not anatomically separate parts, the surgeon must distinguish these parts when planning the surgical treatment of rectal cancer. When viewed with a rigid rectoscope, from the anal verge, these three parts are defined as follows: the lower

Address for Correspondence/Yazışma Adresi: Mehmet Ayhan Kuzu MD, 
rectum, 0 to $7 \mathrm{~cm}$; the middle rectum, 7 to $12 \mathrm{~cm}$; and the upper rectum, beyond $12 \mathrm{~cm}$. However, the length of the rectum may vary individually and may exceed $15 \mathrm{~cm}$. The rectum is mostly extraperitoneal. However, the upper rectum is covered with a thin layer of visceral peritoneum at the anterior and lateral sides. This peritoneal layer extends downward and anteriorly, creating a peritoneal reflection in the anterior of the rectum. Peritoneal reflection corresponds to the level of middle Houston's valve. When dealing with rectal cancer surgery, the intestine and its mesentery, and perimesenteric planes should be removed as a block for surgical principles. In this context, the anatomy of the pelvic structures was divided into six regions for surgeons in order to understand the anatomy. ${ }^{1,2,3,4,5}$ These six regions include:

\section{Above the Pelvic Inlet}

There is a thin facial sheath covering the inferior mesenteric artery, vein, and lymphatics. This sheath is centrally located in the anterior of the preaortic nerve plexuses. Laterally, it is close to the ureter and gonadal vessels. In complex pelvic surgery, the ureter may need to be fully mobilized up to the bladder (Figures 1, 2).

\section{Below Aortic Bifurcation}

The mesorectum, distal to the upper $1 / 3$ rectum, is closely associated with hypogastric nerve bifurcation and presacral adipose tissue (Figures 3,4 ). As the rectum is covered with peritoneum at the anterior, it is adjacent to the small bowel loops, bladder, and uterus.

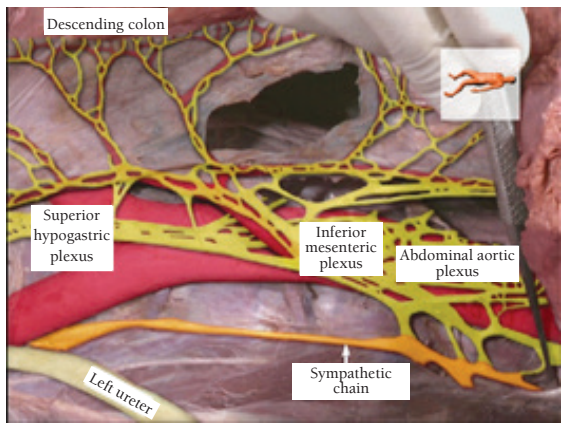

Figure 1. Above the pelvic inlet

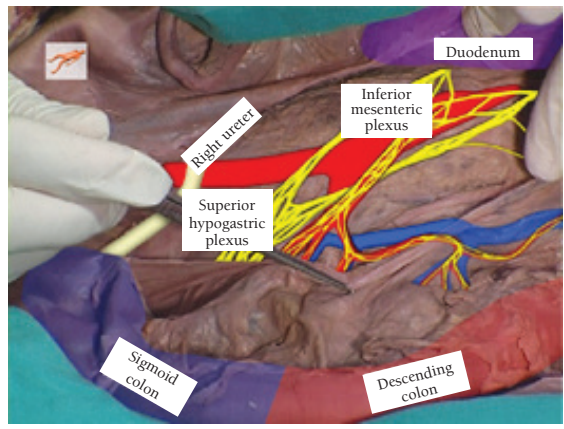

Figure 2. Above the pelvic inlet

\section{Pelvic Side Wall}

In this area, internal iliac vessels, piriformis muscle, fascia, and autonomic nerves covering these structures are found. The distance between the visceral leaf of the endopelvic fascia surrounding the mesorectum (fascia propria recti) and the autonomic nerves is $2-3 \mathrm{~mm}$. More distally, the splanchnic nerve (erigent nerves), often originating from the S3 root of the sacral plexus, joins the hypogastric nerve and together form the inferior hypogastric plexus (Figure 5). The inferior hypogastric plexus is mostly adjacent to the small vessels. If these veins are thick enough, they may take the name of middle rectal veins. However, anatomical dissection studies of Sato showed that the incidence of the middle rectal artery is $22 \%$, and according to the same studies, the middle rectal artery is often unilateral. The width of this artery is $2 \mathrm{~mm}$

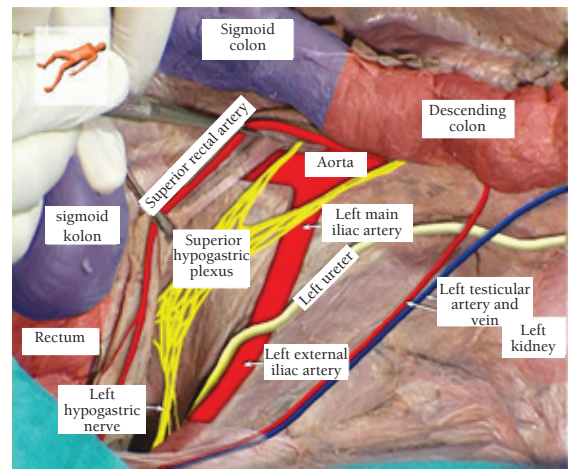

Figure 3. Below aortic bifurcation

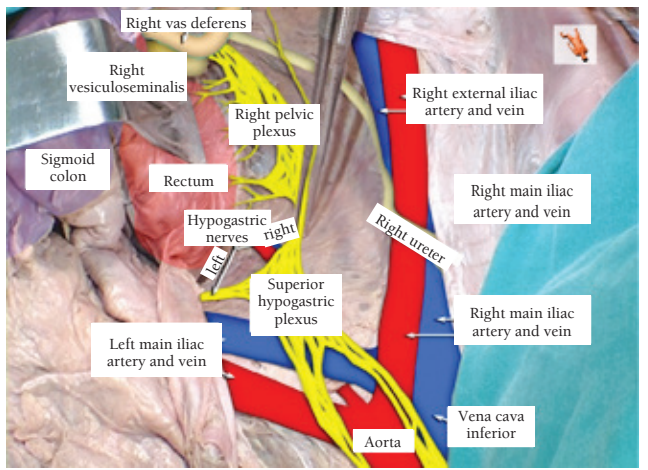

Figure 4. Below aortic bifurcation

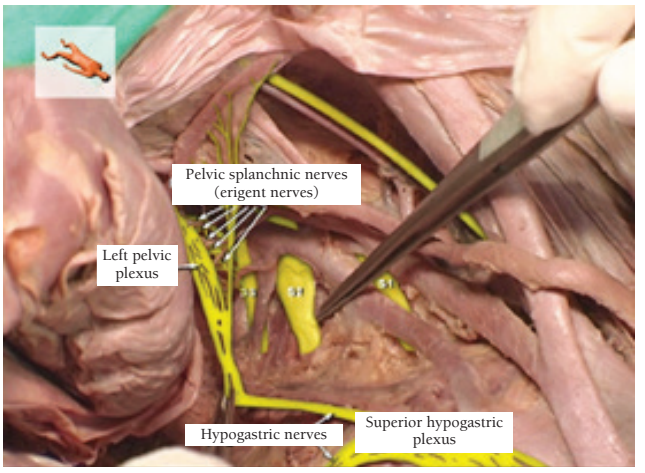

Figure 5. Important nerve structures of the pelvic sidewall 
and can be cut with scissors or cautery without causing significant bleeding when dissecting. It rarely needs ligation. Therefore, as in the past, cutting the lateral ligaments of the rectum with forceps is no longer performed because it causes autonomic nerve damage rather than arterial control. Therefore, progression by visual dissection in the avascular plane is essential for the protection of the autonomic nerves.

\section{Anterior Dissection Area}

The middle rectum is surrounded by the Denonvilliers' fascia in the anterior aspect, and this fascia is located behind the seminal vesicles in men and behind the vagina in women. In men, this fascia progresses very closely to the posterior aspect of the prostate. Therefore, this region may cause severe problems in lower rectal dissections in men.

\section{Posterior Dissection Area}

Distally, the mesorectum is divided into two fatty lobes, and a pit known as anococcygeal raphe remains in the middle (Figure 6). This structure is likened to a "bipolar lipoma" (Figure 8). While both lobes extend a little lower, they show proximity to the pelvic autonomic nerves on the lateral wall due to the narrowing pelvis. Due to this convergence, autonomic nerve injury may occur during dissection.

\section{Intersphincteric Dissection Area}

While the rectum proceeds to the intersphincteric plane at the level of the pelvic floor, the rectum wall continues with the smooth muscles of the internal sphincter. At this level, the mesorectum becomes now very thin, and thus the avascular dissection plane (Holy plane) ends in the intersphincteric plane between the internal and external sphincters. This plane is important in coloanal anastomoses.

\section{Vessels}

\section{Inferior Mesenteric Artery}

The inferior mesenteric artery (IMA) emerges from the anterior aspect of the aorta and moves down to the left at the level of lumbar 2-3, 3-5 cm above the aortic bifurcation. IMA gives rise to the left colic artery and sigmoidal artery branches in the abdomen, which may vary between 2-6 branches. The left colic artery is the first branch of the IMA. The IMA is called the superior hemorrhoidal artery or superior rectal artery after crossing the left iliac artery. The superior hemorrhoidal artery proceeds down to S3 in the sigmoid mesocolon and then down the posterior side of the rectum. In $80 \%$ of the cases, it is divided into two terminal branches, right and left. These branches advance down the rectum submucosa and feed the lower rectum and anal canal. In addition to the superior hemorrhoidal artery, middle, inferior rectal (hemorrhoidal) and median sacral arteries are involved in arterial feeding of the rectum and anal canal.
While the superior rectal artery is the continuation of the IMA, the middle rectal artery is the branch of the internal iliac artery. However, it is assumed that the middle rectal artery is not present in everybody (22\% of the middle rectal arteries could be detected in the autopsy study) and is rarely seen, especially in women. The inferior rectal artery emerges from the internal pudendal artery and provides blood to the area from the anal canal to the pectinate line. The median sacral artery emerges just above the aortic bifurcation, extends downward in front of the lower lumbar vertebrae, sacrum, and coccyx, giving many thin branches to the posterior wall of the rectum (Figures 7, 8, 9). Although considered as a vascular dissection plane, these thin branches can be seen in the mesorectal dissection plane. ${ }^{6,7,8,9,10,11}$

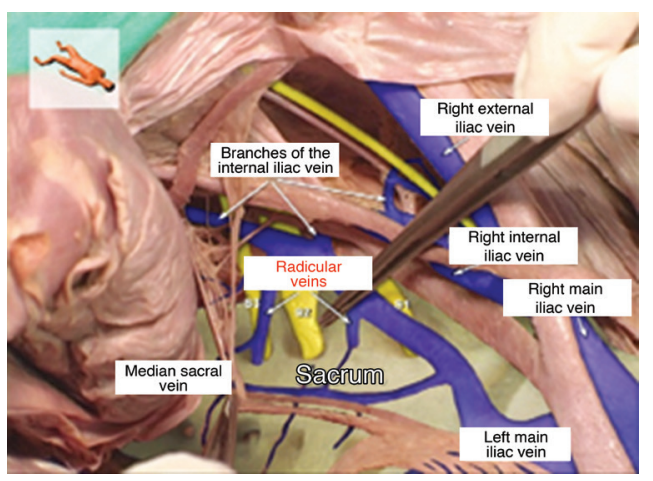

Figure 6. Posterior dissection wall

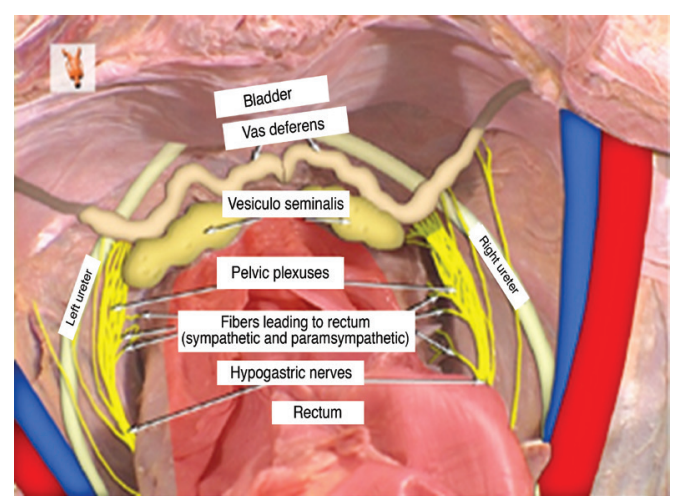

Figure 7. Important arteries and veins of the pelvic and anorectal regions

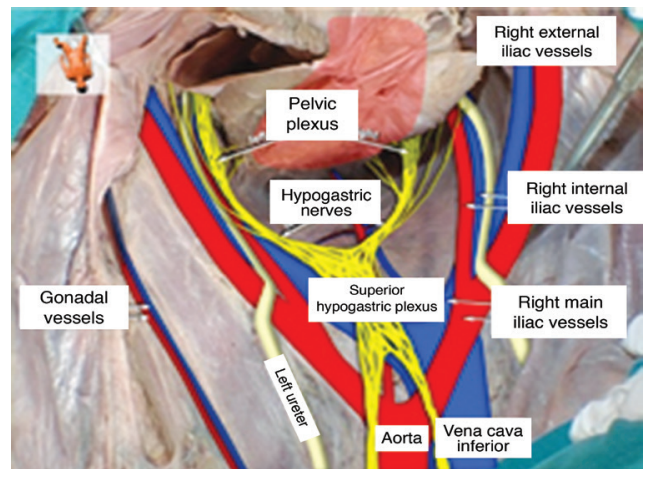

Figure 8. Blood supply of the pelvic and anorectal regions 


\section{Venous Drainage}

The superior rectal vein draining the rectum is poured into the inferior mesenteric vein (IMV). IMV connects with splenic vein and superior mesenteric vein to form the portal vein. Middle and inferior rectal veins are poured into the internal iliac vein and therefore drained into the caval system (Figure 10). Therefore, cancers located in the lower $2 / 3$ of the rectum have the potential to direct lung metastasis through iliac veins without liver metastasis. In this context, unlike colon cancer, lung metastasis rate is higher in rectal cancers. ${ }^{6,7,8,9,10,11}$

\section{Lymphatic Drainage}

The lymphatics of the rectum and anal canal form two extramural plexuses. One is located above, and one is located below the pectinate (dentate) line. The lymphatic drainage at the superior of the pectinate line proceeds backward and along the superior rectal artery, following the artery tracing to the root of the aorta. Also, drainage into the lateral pelvic lymph nodes is observed. In the inferior part of the pectinate (dentate) line, the spread is along with the inguinal lymph nodes. ${ }^{12,13}$

\section{Nerves}

Sympathetic nerves of the left colon and rectum emerge from L1-3, and the preganglionic fibers synapse in the preaortic plexus via the lumbar sympathetic nerves.

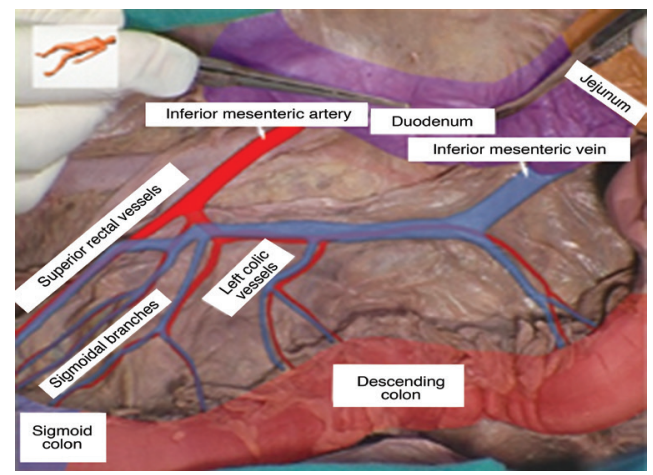

Figure 9. Blood supply of the pelvic and anorectal regions

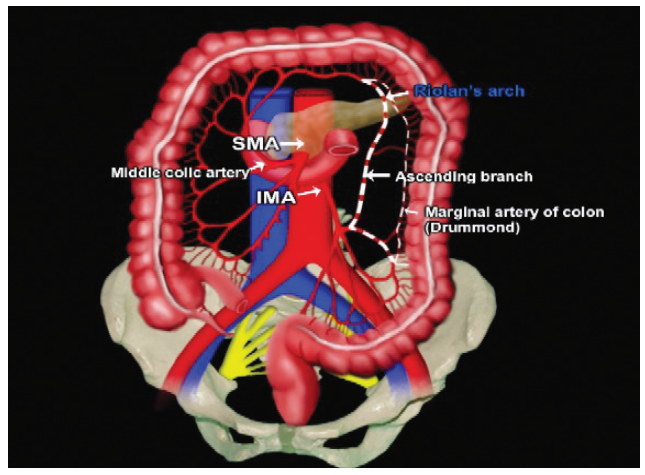

Figure 10. Veins of the pelvic and anorectal regions
Postganglionic fibers innervate the IMA feeding site and the upper part of the rectum. Presacral nerves formed by the combination of aortic plexus and lumbar splanchnic nerves innervate the lower part of the rectum. Presacral nerves form hypogastric plexus just below the sacral promontory. Anal hypogastric nerves on both sides of the rectum carry sympathetic innervation from the hypogastric plexus to the pelvic plexus. The pelvic plexus is located in the lateral part of the pelvis near the lateral ligaments at the level of the lower $1 / 3$ rectum. The parasympathetic innervation of the rectum comes from the S2-4 splanchnic nerves. These fibers originating from the sacral foramen are called erigent nerves. These fibers advance laterally and upward to join the sympathetic hypogastric nerves in the pelvic plexus. Postganglionic parasympathetic and sympathetic fibers originating from the pelvic plexus reach the left colon and upper rectum via the inferior mesenteric plexus. They reach the upper rectal and anal canal directly. Periprostatic plexus, which is the lower part of the pelvic plexus, is located in the Denonvilliers' fascia and innervates the prostate, seminal vesicle, corpus cavernosum, vas deferens, urethra, ejaculatory canals, bulbourethral glands (Figure 11). Cutting of both superior hypogastric plexus or hypogastric nerve causes sympathetic denervation. If the erigent nerves are intact, bladder sphincter dysfunction results in retrograde ejaculation and erectile dysfunction. Dissection near the seminal vesicle and prostate margin may cause damage to the periprostatic plexus, resulting in sympathetic and parasympathetic damage. As a result, erectile dysfunction occurs. One of the main objectives of rectal surgery is to preserve organ functions as much as possible. Therefore, the localization of autonomic nerves according to the surgical dissection plane should be well known. While the left colon is released from the lateral peritoneum, the mesorectal dissection area is entered thorough passing first the gonadal vessels, then the ureter, and then the hypogastric nerve. One should perform sharp dissection by seeing in the mesorectal dissection area. During this dissection, the right and left hypogastric nerves are seen and preserved. In this dissection,

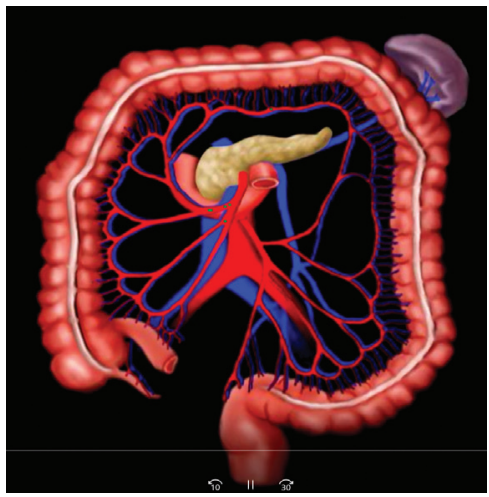

Figure 11. Pelvic plexus and structures innervated by pelvic plexus 
the hypogastric nerves are located in the avascular loose connective tissue between the presacral fascia and the fascia propria recti surrounding the mesorectum, and on the pelvic sidewalls, they are parallel to the ureter. With a tampon placed here, the anatomic structures and nerves can be protected. By retracting the descending colon laterally to the left, a window is opened on the right side of the sigmoid mesocolon, and the previously detected hypogastric nerves can be preserved. Autonomic nerve fibers, which continue on the aorta, form superior hypogastric plexus just above the promontory below the aortic bifurcation. The superior hypogastric plexus is divided into two at the pelvis inlet (wishbone appearance). The right and left hypogastric nerves then form. While entering the mesorectal dissection plane in the pelvic inlet, injuries to the right and left hypogastric nerves may occur. When the peritoneum is opened, if the nerves are not followed from above, the possibility of injury increases. The hypogastric nerves lie parallel to the ureter and iliac artery in the caudal and lateral directions within the mesorectal plane lateral to the rectum. There are autonomic nerve branches from the rectum during this course. While the hypogastric nerves proceed caudally and laterally in the lateral wall of the pelvis, they combine with parasympathetic nerve fibers (erigent nerves) originating from the sacral 2-3$4^{\text {th }}$ roots and the fibers from the pelvic sympathetic trunk and form the inferior hypogastric plexus. The thickest of the parasympathetic fibers from the sacral roots is the one originating from S3. Erigent nerves emerge from the sacral foramen and travel beneath the endopelvic fascia. Very rarely, during the mesorectal dissection, Waldeyer's fascia may be opened (retrorectal area is entered), and erigent nerves can be visualized..$^{14,15}$ In their anatomical studies, Walsh and Danker showed that the vessel nerve package, including pelvic autonomic nerve fibers, was observed at 10 and 2 o'clock at the edges of the seminal vesicles. In women, this vessel nerve package is located in the cardinal ligament in the front of the rectogenital fascia. ${ }^{16}$ In their autopsy and surgical specimen studies, Yamakoski et al. showed that the distance between the rectal muscle layer and pelvic autonomic nerves was approximately $10 \mathrm{~mm}$. They emphasized that the preservation of autonomic nerves may reduce the reliability of lateral resection margins in a cancer surgery performed by preserving the pelvic autonomic nerves. Autonomic nerve damage during rectal cancer surgery can happen at four critical sites: The first of these develops when the root of the IMA is ligated close to the aorta. Dissection and ligation very close to the aorta may cause damage to the aortic plexus. Second, the nerve may be damaged when entering the mesorectal dissection plane or dissection from the posterior. Hypogastric nerve damage may occur if the avascular plane is left during dissection or if the dissection is performed bluntly by hand.
Due to damage of the sympathetic fibers in injuries located cranial to the joining of the parasympathetic fibers in the hypogastric plexus, ejaculation disorders are observed in men, whereas women do not have significant sexual dysfunction. The third most common site of injury to the autonomic nerves is the injury of the inferior hypogastric plexus formed by the junction of the sacral parasympathetic fibers with the hypogastric nerve. This type of injury can occur during lateral mesorectal dissection. In the past, this type of damage was seen frequently because of the ligation or clamping performed to control the lateral ligaments. Nowadays, it is assumed that the rectum does not have lateral ligaments. It has been shown that it consists only of branches entering the rectal artery and middle rectal artery and autonomic nerves. Therefore, lateral dissection should be performed visually, and clamping should not be used. Also, excessive rectal traction during mesorectal dissection may cause such nerve damage. Another injury site of the nerve occurs during anterior dissection of the rectum. Invisible cavernous nerve damage may occur in the dissection between the seminal vesicles and prostate with Denonvilliers' fascia. To prevent cavernous nerve damage, the Denonvilliers' fascia should be dissected proximally before reaching the base of the prostate. Pelvic autonomic nerves, including parasympathetic fibers, are affected when an injury occurs at the third and fourth possible damage sites of the nerve described above. As a result of this, erectile dysfunctions occur in men, and sexual dysfunctions such as decreased vaginal secretion, dyspareunia, lack of orgasm develop in women, and dysfunction of the bladder, including urinary retention or incontinence, may develop in both sexes (Figure 12). Studies have shown that the location of the tumor in the rectum is an essential factor for autonomic nerve injury. For example, nerve damage is higher in distal rectum tumors than in upper rectal tumors, whereas nerve damage may develop more in anterior tumors than posterior tumors. In case of any invasion, oncologically, the autonomic nerves on the invaded side should be resected without caring for an injury. ${ }^{17,18,19}$

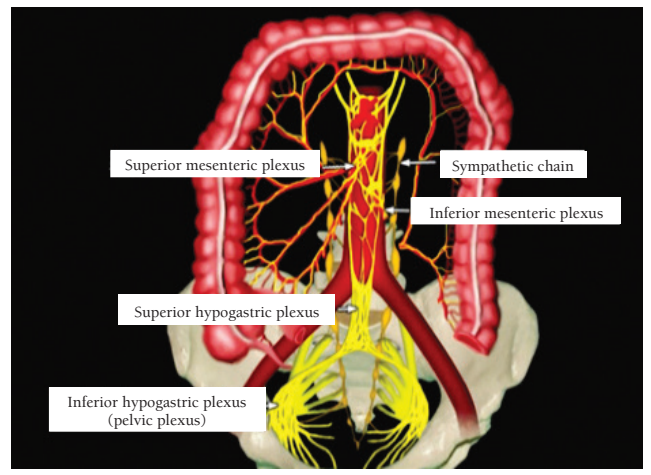

Figure 12. Innervation of the pelvic and anorectal regions 


\section{Ethic}

Peer-review: Internal peer-reviewed.

\section{Authorship Contributions}

Surgical and Medical Practices: H.İ.A., MAK, Concept: H.İ.A., MAK, Design: H.I.A., MAK, Data Collection or Processing: H.I.A., MAK, Analysis or Interpretation: H.I.A., MAK, Literature Search: H.İ.A., MAK, Writing: H.İ.A., MAK, Conflict of Interest: No conflict of interest was declared by the authors.

Financial Disclosure: The authors declared that this study has received no financial support.

\section{References}

1. Chapuis P, Bokey L, Fahrer M, Sinclair G, Bogduk N. Mobilization of the rectum: anatomic concepts and the bookshelf revisited. Dis Colon Rectum 2002;45:1-8.

2. Salerno G, Sinnatamby C, Branagan G, Daniels IR, Heald RJ, Moran BJ. Defining the rectum:surgically, radiologically and anatomically. Colorectal Dis 2006;8:5-9.

3. Dujovny N, Quiros RM, Saclarides TJ. Anorectal anatomy and embryology. Surg Oncol Clin N Am 2004;13:277-293.

4. B.G.A. Moynihan, The surgical treatment of cancer of the sigmoid flexure and rectum, Surg Gynecol Obstet 463 (1908).

5. Miles WE. A method of performing abdomino-perineal excission for carcinoma of the rectum and terminal portion of the pelvic colon (1908) CA Cancer J Clin 1971;21:361-364.

6. Boxall TA, Smart PJG, Griffiths JD. The blood-supply of the distal segment of the rectum in anterior resection. Br J Surg 1963;50:399-404.

7. Crapp AR, Cuthbertson AM. William Waldeyer and the rectosacral fascia Surg Gynecol Obstet 1974;138:252-256.
8. Tobin CE, Benjamin JA. Anatomical and surgical restudy of Denonvilliers' fascia. Surg Gynecol Obstet 1945;80:373-388.

9. Ayoub SF. Arterial supply of the human rectum. Acta Anat 1978;100:317327.

10. Drummond H. The arterial supply of the rectum and pelvic colon. Br J Surg 1914;1:677-685.

11. Sonneland J, Anson BJ, Beaton LE. Surgical anatomy of the arterial supply to the colon from the superior mesenteric artery based upon a study of 600 specimens. Surg Gynecol Obstet 1958;106:385-398.

12. Miscusi G, Masoni L, Dell'Anna A, Montori A. Normal lymphatic drainage of the rectum and the anal canal revealed by lymphoscintigraphy. Coloproctology 1987;9:171-174.

13. Jameson JK, Dobson JF. The lymphatics of the colon. Proc R Soc Med 1909;2:149-172.

14. Heald RJ, Moran BJ. Embryology and Anatomy of the Rectum Seminars in Surgical Oncology 1998; 15:66-71.

15. Clausen N, Wolloscheck T, Moritz A. How to Optimize Autonomic Nerve Preservation in Total Mesorectal Excision: Clinical Topography and Morphology of Pelvic Nerves and Fasciae. World J Surg 2008;32:17681775.

16. Walsh PC, Donker PJ. Impotence following radical prostatectomy: insight into etiology and prevention. 1982. J Urol 2002;167:1005-1010.

17. Heald RJ, Moran BJ, Brown G, Daniels IR. Optimal total mesorectal excision for rectal cancer is by dissection in front of Denonvilliers' fascia British Journal of Surgery 2004;91:121-123.

18. Muntean V. The surgical anatomy of the fasciae and the fascial spaces related to the rectum. Surg Radiol Anat. 1999;21:319-324.

19. Church JM, Raudkivi PJ, Hill GL. The surgical anatomy of the rectum a review with particular relevance to the hazards of rectal mobilisation. Int J Colorectal Dis 1987;2:158-166. 